\title{
28 Research Square \\ Identification of Prognostic Biomarkers of Ewing \\ Sarcoma using Bioinformatics Analysis and Experiments
}

\section{Jun Zhang}

Guangxi Medical University First Affiliated Hospital

\section{Mingwei He}

Guangxi Medical University First Affiliated Hospital

\section{Wenyu Feng}

Guangxi Medical University First Affiliated Hospital

Yun Liu

Guangxi Medical University First Affiliated Hospital

Xiaoting Luo

Guangxi Medical University First Affiliated Hospital

\section{Tianyu Xie}

Guangxi Medical University First Affiliated Hospital

Juliang He

Guangxi Cancer Hospital and Guangxi Medical University Affiliated Cancer Hospital

Jie Ma

Guangxi Medical University First Affiliated Hospital

Qingjun Wei ( $\nabla$ weiqingjungxnn@163.com )

Guangxi Medical University First Affiliated Hospital https://orcid.org/0000-0003-0570-4085

\section{Research}

Keywords: Ewing sarcoma, bioinformatics analysis, prognosis, biomarker, NOL11

Posted Date: February 1st, 2021

DOl: https://doi.org/10.21203/rs.3.rs-164477/v1

License: (c) (i) This work is licensed under a Creative Commons Attribution 4.0 International License. Read Full License 


\section{Abstract}

Background. Ewing sarcoma (EWS) is the second most familiar bone or soft-tissue sarcoma, making the identification of EWS biomarkers critically important. This study aimed to explore and validate potential prognostic biomarkers in EWS by bioinformatics analysis and experiments.

Methods The microarray dataset GSE119546 was downloaded from the Gene Expression Omnibus (GEO) database. Differentially expressed genes (DEGs) between EWS cells treated with and without Cyclindependent kinase 9 (CDK9) inhibitors were analyzed using $R$ package limma. Next, we performed gene enrichment analysis and constructed a protein-protein interaction (PPI) network. The expression level of the top18 hub genes and the overall survival (OS) in patients with sarcoma were validated using the Oncomine and Gene Expression Profiling Interactive Analysis 2 (GEPIA2) databases. Furthermore, the mRNA expression of Bystin Like (BYSL), Nucleolar Protein 11 (NOL11), and P21-activated protein kinaseinteracting protein 1 (PAK1IP1) was detected by RT-PCR in the EWS cell line (A-673), and the protein expression in EWS tissue was verified by immunohistochemistry (IHC).

Results Our study identified 343 DEGs, which were associated with various cancer-related functions and pathways. A total of three DEGs, including BYSL, NOL11, and PAK1IP1, were identified as hub genes with prognostic values using GEPAI2 and Oncomine. Finally, RT-PCR results showed the mRNA expression level of BYSL, NOL11, and PAK1IP1 in A-673 cells was higher than that in human mesenchymal stem cells. IHC staining revealed a positive expression ratio of NOL11, BYSL, and PAK1IP1 was 63.6\% (14/22), $31.9 \%(7 / 22)$, and $0 \%(0 / 22)$, respectively. Cox regression analysis confirmed NOL 11 was a significant prognostic factor for OS.

Conclusions. Our findings revealed that NOL11 may be a potential biomarker for EWS prognosis and treatment.

\section{Background}

Ewing sarcoma (EWS) is a small round cell sarcoma characterized by high aggression and bad outcome [1]. Compared with histological classification, genetic markers serve an increasingly important role in the early diagnosis, treatment, and prognosis of EWS. The main driver of EWS is the reciprocal translocation between the Ewing sarcoma breakpoint region 1 protein (EWSR1) and -Friend leukemia integration 1 transcription factor (FLI1) genes. Many attractive candidate genes, including RE1-silencing transcription factor (REST) [2], NAD-dependent protein deacetylase sirtuin 1 (SIRT1) [3], Nucleosome remodeling deacetylase (NURD) [4] have been detected in EWS. Although these biomarkers play an essential role in Ewing sarcoma progression and prognosis, the majority still had an unfavorable or poor prognosis. The current 5-year overall survival (OS) rate for patients without metastasis is approximately $65-75 \%$, while 5year OS with metastases is lower than $30 \%$ [5]. Thus, there is an urgent demand to explore clinically useful biomarkers for improvements in early diagnosis, OS, and therapeutic strategies. 
CDK9 is a member of the CDK family of serine/threonine kinases present in eukaryotes. It is widely expressed in various sarcomas and contributes to tumor development and progression, such as osteosarcoma [6], EWS [7], and Kaposi's sarcoma [8]. Studies have demonstrated that dysregulation of the CDK9 signaling system may be essential for the occurrence and maintenance of malignant cell phenotypes, such as neuroblastoma [9], prostate cancer[10], and hepatocellular carcinoma [11]. A new promising CDK9 inhibitor (CDKI)-73 downregulated EWS-FLI1 Expression rapidly and blocked the contactdependent proliferation of EWS cells in vitro [12]. Therefore, in this study, the mRNA microarray dataset GSE119546[12] including EWS cells treated with and without CDKI-73, was analyzed using the limma package in RStudio.

Bioinformatics analysis uses mathematics, statistics, and computer science to process and analyze laboratory data and has played a vital role in cancer research [13]. In the current study, we downloaded the mRNA microarray dataset GSE119546 [12] from the GEO database to perform bioinformatics analysis. We applied the R package clusterProfiler and ggplot2 to analyze and visualize $\mathrm{GO}$ terms and KEGG pathways of the DEGs. Then we constructed a PPI network and utilized GEPIA2 and Oncomine to select hub genes with prognostic value. Finally, BYSL, NOL11, and PAK1IP1 were chosen for further validation. RT-PCR was performed to test the mRNA level in EWS cells, and IHC was used to examine protein levels in EWS samples. BYSL gene is mapped to chromosome 6P21.1. It is involved in cell adhesion, which contributes to early embryo implantation. A previous study has reported that BYSL is significantly upregulated in human hepatocellular carcinoma specimens compared with adjacent normal tissues [14]. NOL11 gene is mapped to chromosome 17q24.2. Emily F. Freed1 reported NOL11 is implicated in the Pathogenesis of North American Indian Childhood Cirrhosis [15]. PAKIP1 gene is mapped to chromosome 6P24.2. Recent literature has reported that PAKIP1 was identified as a prognostic factor for Rhabdoid Tumors of the Kidney [16]. However, the clinical significance of BYSL, NOL11, and PAK1IP1 in EWS has not been elucidated. Therefore, we evaluated the association between their expression and the prognosis of EWS patients in our study.

\section{Materials And Methods}

\section{Data pre-processing and DEG analysis}

GSE119546 contains six public gene expression profiles, which can be downloaded from the GEO [17] database (http://www.ncbi.nIm.nih.gov/geo; GPL6244 platform). These profiles that included three human Ewing sarcoma cell lines treated with and without CDKI-73 were submitted by Richter in 2018 and updated in 2020[12]. DEGs were screened using the limma package[18] in RStudio with the threshold of the absolute value of logarithmic change $(\log F C)>1$, and a Benjamini-Hochberg false discovery $P<0.05$ was considered of statistical significance. The ggplot2 (version 3.3.1) package in RStudio was used for data visualization.

\section{KEGG and GO enrichment analyses}


KEGG[19] (http://www.genome.jp/kegg/) is an integrated knowledge database for the practical application of genomic cognition and functional interpretation. The GO [20] database is the largest source of information on the functions of genes. ClusterProfiler (version 3.16.0) is an ontology-based R package that provides enrich KEGG and enrich GO for enrichment analyses and gene classification. In the present study, GO terms, and KEGG pathways of the DEGs were analyzed by the "clusterProfiler" package and visualized by the ggplot2 package in RStudio. $P<0.05$ was selected as the threshold for the identification of significant terms and pathways.

\section{PPI network construction and hub genes analyses}

The online biological database STRING [21] (http://string-db.org, version 11.0) was used in the present study to construct a PPI network of the DEGs with statistical significance using the minimum required interaction scores $>0.7$ (high confidence). The co-expression results were exported from the STRING database as a simple tabular text output and imported into Cytoscape [22] software (version 3.7.2) for visualization. cytoHubba [23] is a plug-in of Cytoscape that can sort nodes in a network by their network characteristics. The top 18 hub genes were chosen by cytoHubba based on the "Closeness" algorithm.

\section{Oncomine and GEPIA2 analysis}

The Oncomine database [24](version 4.5; http://www.oncomine.org) facilitates the implementation of collecting, assessing, and transmitting cancer transcriptome data for biomedical researchers. In the current study, Oncomine was applied to explore the mRNA expression levels of crucial genes in sarcoma and normal tissues. The threshold limits were as follows: P-value, 0.05 ; fold-change, 1.5. GEPIA2 [25] (http://gepia2.cancer-pku.cn) is a reinforced website that couples The Cancer Genome Atlas (TCGA) and Genotype-Tissue Expression (GTEx) datasets to provide expression profiling and integrative analysis. The OS analysis of the hub genes in EWS was performed using GEPIA2 single-gene analysis.

\section{Detection of mRNA expression by RT-PCR}

EWS cell line A-673 was obtained from the American Type Culture Collection (ATCC, CRL-1598 ${ }^{\text {TM }}$ ). Human mesenchymal stem cells (hMSCs) were obtained from Beina Chuanglian Biotechnology Research Institute (Beijing, China). The A-673 cells and hMSCs were cultured in Dulbecco's Modified Eagle's Medium (DMEM) with 10\% fetal bovine serum (FBS). Total RNA was extracted from the hMSCs and A673 cells using the TriQuick RNA kit (Beijing, Solarbio) according to the protocol. RNA was converted into cDNA through a reverse transcription reaction accompanied with oligo (dT) primers using PrimeScript ${ }^{\mathrm{TM}}$ RT Master Mix (TaKaRa, Japan). $\beta$-actin was used as the internal control. The primer sequences were listed as follows: NOL11, 5'- TTAGAGGCCTTGCTTGCAGA-3' (forward), and 5'-

AAAACAGGATGTCTGAATACTACGA -3' (reverse);BYSL, 5'- GTCTGGCACTTGTACCCTCC -3' (forward) and 5'- TTGGCACCGCTGTATTCCAT -3' (reverse); PAK1IP1, 5'- CTGCCACAGCCATGCATAAA -3' (forward) and 5'- 
CCTCGGCCTTGAACATTCTCT-3'(reverse); $\beta$-actin, 5'-GTCATTCCAAATATGAGATGCGT-3' (forward) $ه 5$ 'GCTATCACCTCCCCTGTGTG-3' (reverse).

\section{IHC analysis}

Serial sections of paraffin-embedded biopsy specimens from 22 patients with EWS were provided by the First Affiliated Hospital of Guangxi Medical University with Ethics Committee approval. All patients were informed about the experiments and signed informed consent (2021 KY-E-015). The antibodies used for IHC were anti-NOL11(NBP1-90522, Novus Biologicals, USA), anti-BYSL (NBP1-89501, Novus Biologicals, USA), and anti-PAK1IP1 (NBP1-90029, Novus Biologicals, USA). The IHC staining protocol followed the manufactures' instructions. Two pathologists complied with the following criteria to count the average ratio of positive EWS cells: 1 (1-25\%); 2 (26-50\%); $3(51-75 \%)$; and $4(76-100 \%)$. The degree of the staining was scored as follows: 0 (negative); 1 (weak); 2 (medium) and 3 (strong). The ultimate scores coupled ratio and staining degree scores. Samples with a sum of immunoreactive scores (IRS) $\geq 1$ [26]were regarded as positive for NOL11, BYSL, and PAK1IP1.

\section{Statistical analyses}

RStudio (Version 1.2.5042) was used for data analyses. The difference between groups was assessed by the student's t-test. We performed the Kaplan-Meier method to estimate OS curves. Fisher's exact test was performed to evaluate the relationship between hub genes expression and clinical characteristics of patients with EWS. Cox regression analyses were employed to ascertain prognostic factors for OS. $P$ values, $<0.05$ were considered significant.

\section{Results}

\section{Identification Of Degs In Ews}

The medians of the GSE119546 gene expression profiles exhibited inconsistency prior to normalization (Fig. 1a), whereas the median displayed an acceptable level of consistency after normalization (Fig. 1b). A volcano plot was constructed using the ggplot2 package in $R$ for visualization (Fig. 1c). A total of 23,307 genes were involved in the GSE119546 dataset, and 343 genes were determined to be DEGs. Of the 343 genes, 85 genes were upregulated, and 258 were downregulated.

\section{Go And Kegg Enrichment Analyses}

To ensure that crucial enrichment pathways are not lost, upregulated and downregulated genes were analyzed together. GO terms analysis comprised biological process (BP; Fig. 1d), cellular component (CC; Fig. 1e), and molecular function (MF; Fig. 1f) terms. Among the KEGG pathways, the DEGs were 
associated with "herpes simplex virus infection," "RNA transport," "cell cycle," "breast cancer," and "small cell lung cancer" (Fig. 1g). BP analysis revealed that "ribonucleoprotein complex biogenesis," "ncRNA processing," and "mRNA processing" were mainly enriched with DEGs. CC analysis demonstrated that the

"Sm-like protein family complex," "spliceosomal snRNP complex," and "transcription factor complex" were primarily enriched with DEGs. In MF analysis, "catalytic activity," "DNA-binding transcription activator activity," and "transcription coregulator activity" were mostly enriched with DEGs.

\section{Selection Of The Most Important Modules}

The backbone network of DEGs is comprised of 109 nodes and 337 edges (Fig. 1h). A total of 18 genes, including LYAR, PLK1, TFB2M, DIEXF, UTP15, BRIX1, RIOK2, RPS14, NGDN, MPHOSPH10, NOL11, BYSL, LTV1, RPP14, RPP38, NIFK, RRS1, and PAK1IP1 were selected as hub genes using cytoHubba (Fig. 1i).

\section{Validation Of Hub Genes And Survival Analysis}

As presented in Figure. 2A, ten genes, including PLK1, DIEXF, BRIX1, RIOK2, MPHOSPH10, NOL11, BYSL, LTV1, NIFK, and PAK1IP1, were significantly upregulated in sarcoma samples. Next, the survival plots of the hub genes were displayed using the Kaplan-Meier method in GEPIA2 (Figs. 2.B). Patients with low expression levels of BYSL, PAK1IP1, and NOL11 exhibited improved OS with statistical significance.

\section{The mRNA expression of NOL11, BYSL, and PAK1IP1 in A-673 cells}

RT-PCR was performed to detect the mRNA level of NOL11, BYSL, PAK1IP1 in A-673 cells and hMSCs. As presented in Figure.3A, the mRNA expression levels of NOL11, BYSL, PAK1IP1 in the A-673 cells were approximately $4.9,4.7$, and 1.7 times higher than those in the hMSCs, respectively.

\section{The protein expression level and potential value of BYSL and PAK1IP1}

IHC staining revealed the Expression of NOL11, BYSL, and PAK1IP1 proteins in 22 EWS tissues. NOL11, BYSL, and PAK1IP1 positive expression was found in 14 (63.6\%), 7 (31.9\%) and 0 (0\%)patients, respectively. The main staining part of NOL11 (Figs. 3.D-G), BYSL (Figs. 3.H-K), was in the nucleus. Clinicopathological relevance analysis showed NOL11 expression was related to OS, metastasis, and Enneking stage with statistical significance (Table 1). However, the statistical relationship between BYSL expression and clinicopathological characters was not observed except the primary site. A Kaplan-Meier curve indicated that patients with NOL11 positive expression had significantly worse OS than those with NOL11 negative expression (Fig. 3.L). Besides, BYSL did not seem to affect the OS of patients with EWS. ( Fig. 3.M). We performed univariate Cox regression analysis. The results revealed that NOL11, metastasis, and Enneking stage were predictors of OS. However, in multivariate analyses, NOL11 was not an independent prognostic factor for OS ( Table 2).

\section{Discussion}


EWS is a primary malignant bone or soft-tissue sarcoma that mainly occurs in children, adolescents, and young adults [1]. Useful biomarkers are of great significance for informing prognosis and drug discovery. Thus we analyzed the microarray dataset that included human Ewing sarcoma cell lines treated with and without CDK9-inhibitors.

In the present study, a total of 343 genes were identified as DEGs from the GSE119546 dataset, including 85 upregulated and 258 downregulated genes. KEGG pathway and GO term enrichment analyses were performed to explore the MFs, BPs, CCs, and pathways for these DEGs. KEGG pathway analysis results demonstrated that they had been associated with "herpes simplex virus infection," "RNA transport," "breast cancer," "small cell lung cancer," "hepatocellular carcinoma," and "gastric cancer." A previous study has revealed that herpes simplex virus oncolytic treatment based on gene therapy is considered effective in killing EWS cells [27]. RNA transport has been suggested to serve essential roles in the onset of EWS [28]. BP, CC, and MF GO analysis manifested that the DEGs were mainly associated with "ribonucleoprotein complex biogenesis," "Sm-like protein family," and "DNA-binding transcription," respectively. Ribonucleoprotein complex biogenesis induces EWSR1 mutations in EWS [29]. The Sm-like protein family exhibits high expression in pancreatic cancer and several cancer-derived cell lines [30]. Therefore, the results of the KEGG pathway and GO term analyses were consistent with the roles of the DEGs involved in EWS identified in the present study.

In the PPI network, the top 18 genes (LYAR, PLK1, TFB2M, DIEXF, UTP15, BRIX1, RIOK2, RPS14, NGDN, MPHOSPH10, NOL11, BYSL, LTV1, RPP14, RPP38, NIFK, RRS1, and PAK1IP1) that selected from the DEGs were identified as the hub genes. The expression levels of the 18 hub genes were downregulated in EWS cells treated with CDK-173 compared with those in untreated cells. The low expression levels of these hub genes might predict prolonged survival of patients with EWS. Therefore, we performed a prognosis analysis of the 18 hub genes using the GEPIA2 database, and the results revealed that low expression levels of BYSL, NOL11, PAK1IP1 were associated with improved OS in patients with sarcoma. Thus, BYSL, PAK1IP1, and NOL11, which were validated both in the Oncomine and GEPIA2 database, were selected as the candidate genes for the next study.

To further verify the aforementioned bioinformatics analysis, we tested the mRNA expression of BYSL, NOL11, and PAK1IP1 in EWS cells by RT-PCR and examined the protein expression in EWS tissue by IHC. RT-PCR results showed that the mRNA expression levels of NOL11, BYSL, PAK1IP1 in the A-673 cells were higher than those in the hMSCs. IHC staining results revealed that BYSL, NOL11, was positively expressed in EWS tissues. The Expression of NOL11 was associated with OS, metastasis, and the Enneking stage. Patients with high NOL11 expression had a poor prognosis. The Cox model suggested that high NOL11 expression is a significant risk factor for OS of EWS patients.

NOL11 is a novel nucleolar-associated protein that controls cell cycle progression by participating in nucleolar integrity. Hayashi observed that the deficiency of NOL11 delays cell entry into the mitotic phase by inhibiting CDK1 activation[31]. Oncogenic EWS/ FLI1 protein is also involved in tumorigenesis through cell cycle modulation in EWS [32]. Also, depletion of NOL11 decreases ribosomal RNA levels and results 
in nucleolar disruption during interphase [15]. Based on these reports, NOL11 may play the same role in EWS.

The BYSL gene encodes a protein involved in cell adhesion, which contributes to early embryo implantation. A previous study has reported that BYSL is significantly upregulated in human hepatocellular carcinoma specimens compared with adjacent normal tissues. In vitro, knockdown of BYSL inhibits hepatocellular carcinoma cell proliferation, induces apoptosis, and partially blocks the cell cycle in the G2/M phase [14]. Godoy has reported that immunoexpressions of BYSL are observed in $80 \%$ of ovarian tumors [33]. Our study also revealed that BYSL was detected in EWS tissues, but its expression does not alter the clinical course of EWS. PAK1IP1 has been identified as a nucleolar protein that serves a crucial role in cell growth and proliferation by regulating the p53-MDM2 feedback loop. Interestingly, overexpression and knockdown of PAK1IP1 both inhibit cell proliferation and induce p53-dependent cell cycle arrest at the $\mathrm{G} 1$ phase [34]. However, in our study, the protein expression of PAK1IP1 was not observed in EWS tissue using IHC staining. This may be due to the dysregulation of mRNA translation and energy metabolism in cancer [35] or degradation of the protein.

\section{Conclusion}

In summary, we combined bioinformatics methods, RT-PCR, and IHC to explore and verify the expression and clinical significance of hub genes. We observed that NOL11 was positively expressed in EWS cells and tissues. The increased Expression of NOL11 contributes to poor EWS prognosis. Therefore, NOL11 may be a useful biomarker for prognosis evaluation and therapy in EWS.

\section{Abbreviations}

EWS, Ewing sarcoma; GEO, Gene Expression Omnibus; DEGs differentially expressed genes; CDK9, Cyclin-dependent kinase 9; OS, overall survival; GEPIA2, Gene Expression Profiling Interactive Analysis 2; PPI, protein-protein interaction; GO, Gene Ontology; KEGG, Kyoto Encyclopedia of Genes and Genomes; BP, biological process; CC, cellular component; MF, molecular function; BYSL, Bystin Like; NOL11, Nucleolar Protein 11; PAK1IP1, P21-activated protein kinaseinteracting protein 1; TCGA, The Cancer Genome Atlas; IHC, immunohistochemistry; hMSCs, human mesenchymal stem cells

\section{Declarations}

\section{Acknowledgments}

None.

CRediT authorship contribution statement 
Junlei Zhang: Conceptualization, Methodology, Investigation, Writing an original draft. Mingwei He: Data curation, Software, Validation. Wenyu Feng: Validation. Yun Liu: Visualization. Xiaoting Luo: Data curation. Tianyu Xie: Software Juliang He: Methodology. Jie Ma: Supervision, Revising, Project administration. Qingjun Wei: Supervision, Revising, Project administration.

\section{Informed consent}

All patients in this study signed tissue sample consent for clinical research.

\section{Funding}

This study was supported by the Guangxi Science and Technology Plan Project (No. AB17292073).

\section{Availability of data and materials}

The dataset(s) supporting the conclusions of this article is(are) available in the GEO database. https://www.ncbi.nlm.nih.gov/geo/query/acc.cgi?acc=GSE119546

\section{Ethics approval and consent to participate}

This study was approved by the Ethics Committee of the First Affiliated Hospital of Guangxi Medical University, Nanning, China (2021 KY-E-015).

\section{Consent for publication}

Not applicable.

\section{Conflict of interest}

The authors announce that they do not have any conflicting interests.

\section{References}

1. Gaspar N, Hawkins DS, Dirksen U, Lewis IJ, Ferrari S, Le Deley MC, et al. Ewing Sarcoma: Current Management and Future Approaches Through Collaboration. J Clin Oncol. 2015;33(27):3036-46.

2. Ooi L, Wood IC. Chromatin crosstalk in development and disease: lessons from REST. Nature reviews Genetics. 2007;8(7):544-54.

3. Ban J, Aryee DN, Fourtouna A, van der Ent W, Kauer M, Niedan S, et al. Suppression of deacetylase SIRT1 mediates tumor-suppressive NOTCH response and offers a novel treatment option in metastatic Ewing sarcoma. Cancer research. 2014;74(22):6578-88.

4. Sankar S, Bell R, Stephens B, Zhuo R, Sharma S, Bearss DJ, et al. Mechanism and relevance of EWS/FLI-mediated transcriptional repression in Ewing sarcoma. Oncogene. 2016;35(47):6155-6. 
5. Grunewald TGP, Cidre-Aranaz F, Surdez D, Tomazou EM, de Alava E, Kovar H, et al. Ewing sarcoma. Nat Rev Dis Primers. 2018;4(1):5.

6. Ma H, Seebacher NA, Hornicek FJ, Duan Z. Cyclin-dependent kinase 9 (CDK9) is a novel prognostic marker and therapeutic target in osteosarcoma. EBioMedicine. 2019;39:182-93.

7. Flores G, Everett JH, Boguslawski EA, Oswald BM, Madaj ZB, Beddows I, et al. CDK9 Blockade Exploits Context-dependent Transcriptional Changes to Improve Activity and Limit Toxicity of Mithramycin for Ewing Sarcoma. Mol Cancer Ther. 2020;19(5):1183-96.

8. Chang PC, Li M. Kaposi's sarcoma-associated herpesvirus K-cyclin interacts with Cdk9 and stimulates Cdk9-mediated phosphorylation of p53 tumor suppressor. Journal of virology. 2008;82(1):278-90.

9. De Falco G, Bellan C, D'Amuri A, Angeloni G, Leucci E, Giordano A, et al. Cdk9 regulates neural differentiation and its expression correlates with the differentiation grade of neuroblastoma and PNET tumors. Cancer Biol Ther. 2005;4(3):277-81.

10. Lee DK, Duan HO, Chang C. Androgen receptor interacts with the positive elongation factor P-TEFb and enhances the efficiency of transcriptional elongation. J Biol Chem. 2001;276(13):9978-84.

11. Huang CH, Lujambio A, Zuber J, Tschaharganeh DF, Doran MG, Evans MJ, et al. CDK9-mediated transcription elongation is required for MYC addiction in hepatocellular carcinoma. Genes Dev. 2014;28(16):1800-14.

12. Richter GHS, Hensel T, Schmidt O, Saratov V, von Heyking K, Becker-Dettling F, et al. Combined Inhibition of Epigenetic Readers and Transcription Initiation Targets the EWS-ETS Transcriptional Program in Ewing Sarcoma. Cancers (Basel). 2020;12(2).

13. Tao Z, Shi A, Li R, Wang Y, Wang X, Zhao J. Microarray bioinformatics in cancer- a review. Journal of BUON: official journal of the Balkan Union of Oncology. 2017;22(4):838-43.

14. Wang $\mathrm{H}$, Xiao W, Zhou Q, Chen Y, Yang S, Sheng J, et al. Bystin-like protein is upregulated in hepatocellular carcinoma and required for nucleologenesis in cancer cell proliferation. Cell research. 2009;19(10):1150-64.

15. Freed EF, Prieto JL, McCann KL, McStay B, Baserga SJ. NOL11, implicated in the pathogenesis of North American Indian childhood cirrhosis, is required for pre-rRNA transcription and processing. PLoS Genet. 2012;8(8):e1002892.

16. Wang X, Wu X, Li T, Cui M, Zhu L, Wang G, et al. Identification of Biomarkers to Construct a Competing Endogenous RNA Network and Establishment of a Genomic-Clinicopathologic Nomogram to Predict Survival for Children with Rhabdoid Tumors of the Kidney. BioMed research international. 2020;2020:5843874.

17. Barrett T, Wilhite SE, Ledoux P, Evangelista C, Kim IF, Tomashevsky M, et al. NCBI GEO: archive for functional genomics data sets-update. Nucleic Acids Res. 2013;41(Database issue):D991-5.

18. Ritchie ME, Phipson B, Wu D, Hu Y, Law CW, Shi W, et al. limma powers differential expression analyses for RNA-sequencing and microarray studies. Nucleic Acids Res. 2015;43(7):e47. 
19. Kanehisa M, Goto S. KEGG: kyoto encyclopedia of genes and genomes. Nucleic Acids Res. 2000;28(1):27-30.

20. Ashburner M, Ball CA, Blake JA, Botstein D, Butler H, Cherry JM, et al. Gene ontology: tool for the unification of biology. The Gene Ontology Consortium. Nat Genet. 2000;25(1):25-9.

21. Szklarczyk D, Franceschini A, Wyder S, Forslund K, Heller D, Huerta-Cepas J, et al. STRING v10: protein-protein interaction networks, integrated over the tree of life. Nucleic Acids Res. 2015;43(Database issue):D447-52.

22. Shannon P, Markiel A, Ozier O, Baliga NS, Wang JT, Ramage D, et al. Cytoscape: a software environment for integrated models of biomolecular interaction networks. Genome research. 2003;13(11):2498-504.

23. Chin $\mathrm{CH}$, Chen $\mathrm{SH}, \mathrm{Wu} \mathrm{HH}, \mathrm{Ho} \mathrm{CW}$, Ko MT, Lin CY. cytoHubba: identifying hub objects and subnetworks from complex interactome. BMC systems biology. 2014;8(Suppl 4):11. Suppl 4(.

24. Rhodes DR, Yu J, Shanker K, Deshpande N, Varambally R, Ghosh D, et al. ONCOMINE: a cancer microarray database and integrated data-mining platform. Neoplasia (New York NY). 2004;6(1):1-6.

25. Tang Z, Kang B, Li C, Chen T, Zhang Z. GEPIA2: an enhanced web server for large-scale expression profiling and interactive analysis. Nucleic Acids Res. 2019;47(W1):W556-W60.

26. Lu W, Luo JY, Wu MH, Hou JY, Yang X, Chen G, et al. Expression of vimentin in nasopharyngeal carcinoma and its possible molecular mechanism: A study based on immunohistochemistry and bioinformatics analysis. Pathol Res Pract. 2019;215(5):1020-32.

27. Bharatan NS, Currier MA, Cripe TP. Differential susceptibility of pediatric sarcoma cells to oncolysis by conditionally replication-competent herpes simplex viruses. J Pediatr Hematol Oncol. 2002;24(6):447-53.

28. Marko M, Vlassis A, Guialis A, Leichter M. Domains involved in TAF15 subcellular localisation: dependence on cell type and ongoing transcription. Gene. 2012;506(2):331-8.

29. Chi B, O'Connell JD, locolano AD, Coady JA, Yu Y, Gangopadhyay J, et al. The neurodegenerative diseases ALS and SMA are linked at the molecular level via the ASC-1 complex. Nucleic Acids Res. 2018;46(22):11939-51.

30. Watson PM, Miller SW, Fraig M, Cole DJ, Watson DK, Boylan AM. CaSm (LSm-1) overexpression in lung cancer and mesothelioma is required for transformed phenotypes. Am J Respir Cell Mol Biol. 2008;38(6):671-8.

31. Hayashi Y, Fujimura A, Kato K, Udagawa R, Hirota T, Kimura K. Nucleolar integrity during interphase supports faithful Cdk1 activation and mitotic entry. Sci Adv. 2018;4(6):eaap7777.

32. Kowalewski AA, Randall RL, Lessnick SL. Cell Cycle Deregulation in Ewing's Sarcoma Pathogenesis. Sarcoma. 2011;2011:598704.

33. Godoy H, Mhawech-Fauceglia P, Beck A, Miliotto A, Miller A, Lele S, et al. Developmentally restricted differentiation antigens are targets for immunotherapy in epithelial ovarian carcinoma. International journal of gynecological pathology: official journal of the International Society of Gynecological Pathologists. 2013;32(6):536-40. 
34. Yu W, Qiu Z, Gao N, Wang L, Cui H, Qian Y, et al. PAK1IP1, a ribosomal stress-induced nucleolar protein, regulates cell proliferation via the p53-MDM2 loop. Nucleic Acids Res. 2011;39(6):2234-48.

35. Leibovitch M, Topisirovic I. Dysregulation of mRNA translation and energy metabolism in cancer. Adv Biol Regul. 2018;67:30-9.

\section{Tables}


Table 1

Clinical characters of patients with EWS and corresponding NOL11 and BYSL immunostaining

NOL11

negative positive P-value

Cha
OS

No

$2(25.0 \%) \quad 12(85.7 \%)$

0.017

$6(75.0 \%) \quad 2(14.3 \%)$

Yes

Gender

female

$3(37.5 \%) \quad 3(21.4 \%)$

0.752

$5(62.5 \%) \quad 11(78.6 \%)$

male

Age(years)

$\geq 16$

$4(50.0 \%)$

$6(42.9 \%)$

1

$8(53.3 \%)$

$2(28.6 \%)$

0.531

$₫ 16$

$4(50.0 \%) \quad 8(57.1 \%)$

$7(46.7 \%) \quad 5(71.4 \%)$

Primary site

Axial

$2(25.0 \%) \quad 8(57.1 \%)$

0.346

$9(60.0 \%)$

$4(26.7 \%)$

$2(13.3 \%)$

$1(12.5 \%) \quad 1(7.1 \%)$

Metastasis

No

6 (75.0\%) $3(21.4 \%)$

0.045

$6(40.0 \%)$

$9(60.0 \%)$

$1(14.3 \%)$

0.034

$2(25.0 \%) \quad 11(78.6 \%)$

Enneking stage

\begin{tabular}{|c|c|c|c|c|c|c|}
\hline 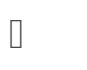 & 6 (75.0\%) & $3(21.4 \%)$ & \multirow[t]{2}{*}{0.045} & 6 (40.0\%) & 3 (42.9\%) & \multirow[t]{2}{*}{1} \\
\hline प & $2(25.0 \%)$ & $11(78.6 \%)$ & & $9(60.0 \%)$ & $4(57.1 \%)$ & \\
\hline \multicolumn{7}{|c|}{ Recurrence } \\
\hline No & $4(50.0 \%)$ & $9(64.3 \%)$ & \multirow[t]{2}{*}{0.838} & $8(53.3 \%)$ & $5(71.4 \%)$ & \multirow[t]{2}{*}{0.735} \\
\hline Yes & $4(50.0 \%)$ & 5 (35.7\%) & & 7 (46.7\%) & $2(28.6 \%)$ & \\
\hline
\end{tabular}

Note: Bold values indicate $\mathrm{P} \otimes 0.05$. OS, overall survival. 
Table 2

Univariate and multivariate Cox models of EWS for NOL11 and BYSL

\begin{tabular}{|lllllll|}
\hline & \multicolumn{3}{c}{ Univariate analysis } & \multicolumn{3}{c|}{ Multivariate analysis } \\
\hline Characteristics & HR & $95 \% \mathrm{Cl}$ & P-Value & HR & $95 \% \mathrm{Cl}$ & P-Value \\
\hline NOL11 & 7.07 & $1.55-32.31$ & 0.01167 & 1.68 & $0.27-10.42$ & 0.577 \\
BYSL & 0.8 & $0.25-2.57$ & 0.70628 & & & \\
Gender & 0.9 & $0.25-3.25$ & 0.86931 & & & \\
Age & 1.67 & $0.57-4.91$ & 0.35349 & 1.94 & $0.54-7.03$ & 0.311 \\
\hline Primary site & 0.8 & $0.32-1.98$ & 0.50043 & & & \\
\hline Metastasis & 13.54 & $1.73-105.78$ & 0.01298 & 9.95 & $0.82-120.7$ & 0.071 \\
\hline Enneking stage & 13.54 & $1.73-105.78$ & 0.01298 & & & \\
\hline Recurrence & 0.61 & $0.2-1.86$ & 0.38352 & 0.4 & $0.1-1.62$ & 0.199 \\
\hline Note: Bold values indicate P凶0.05. HR, hazard ratio; Cl, confidence interval. & \\
\hline
\end{tabular}

Figures 
A

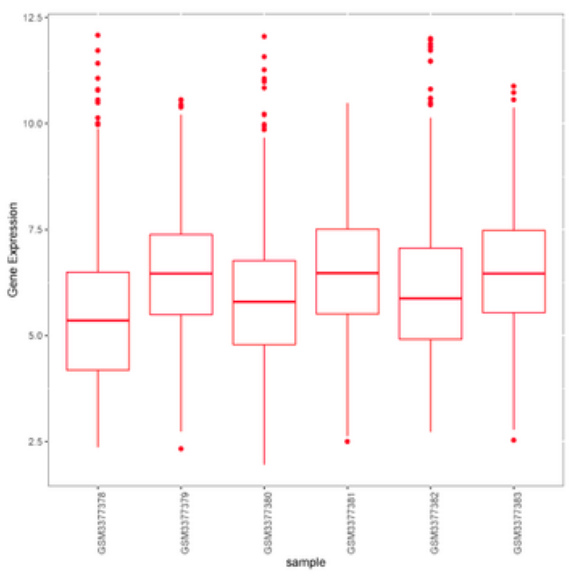

B

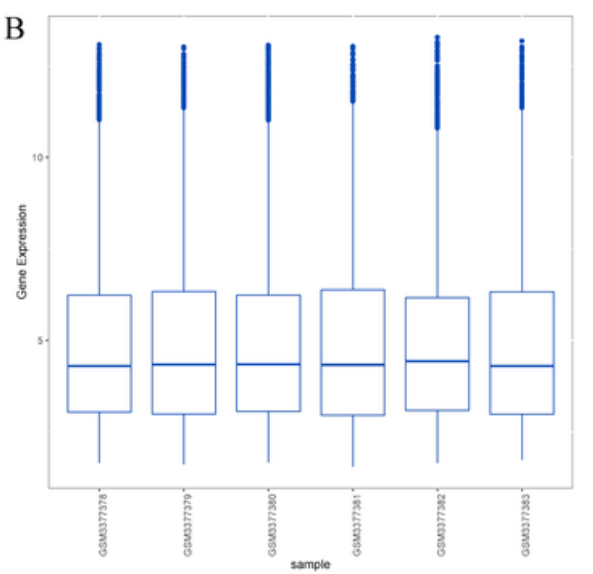

$\mathrm{C}$

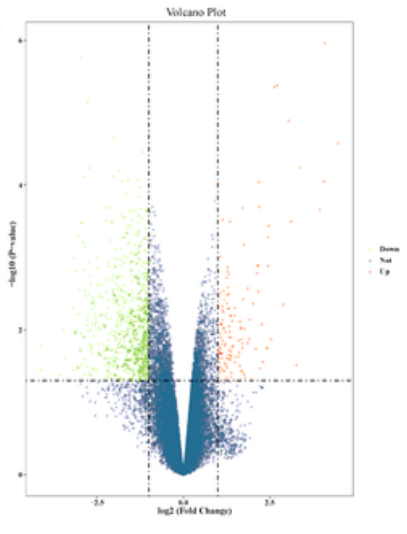

$\mathrm{D}$

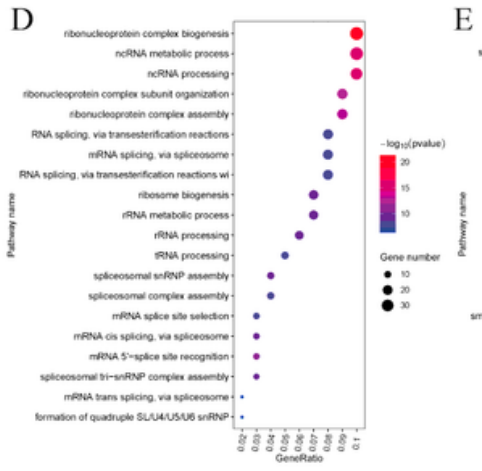

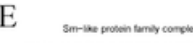
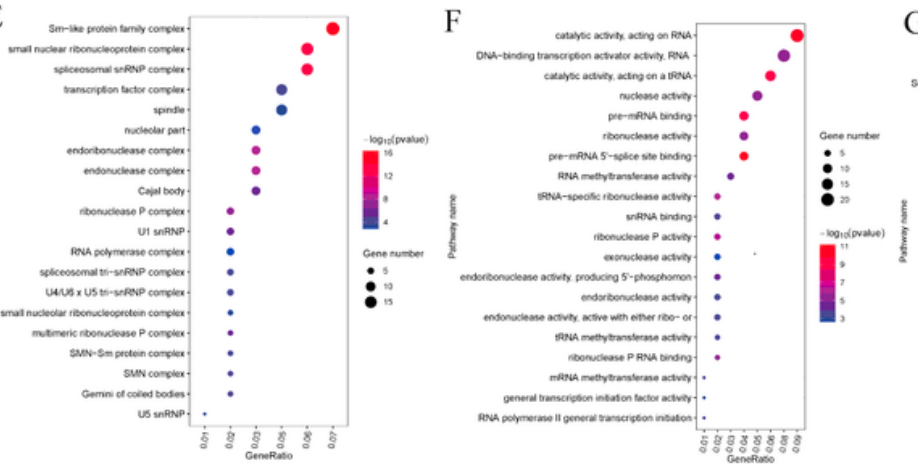

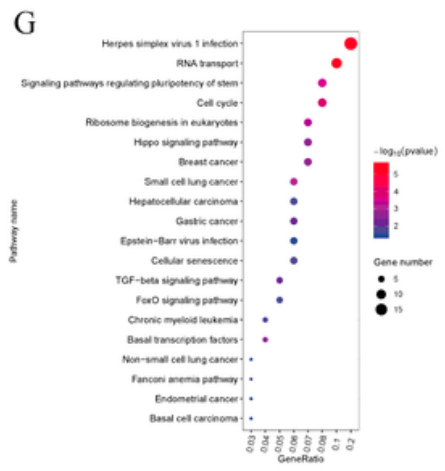

$\mathrm{H}$
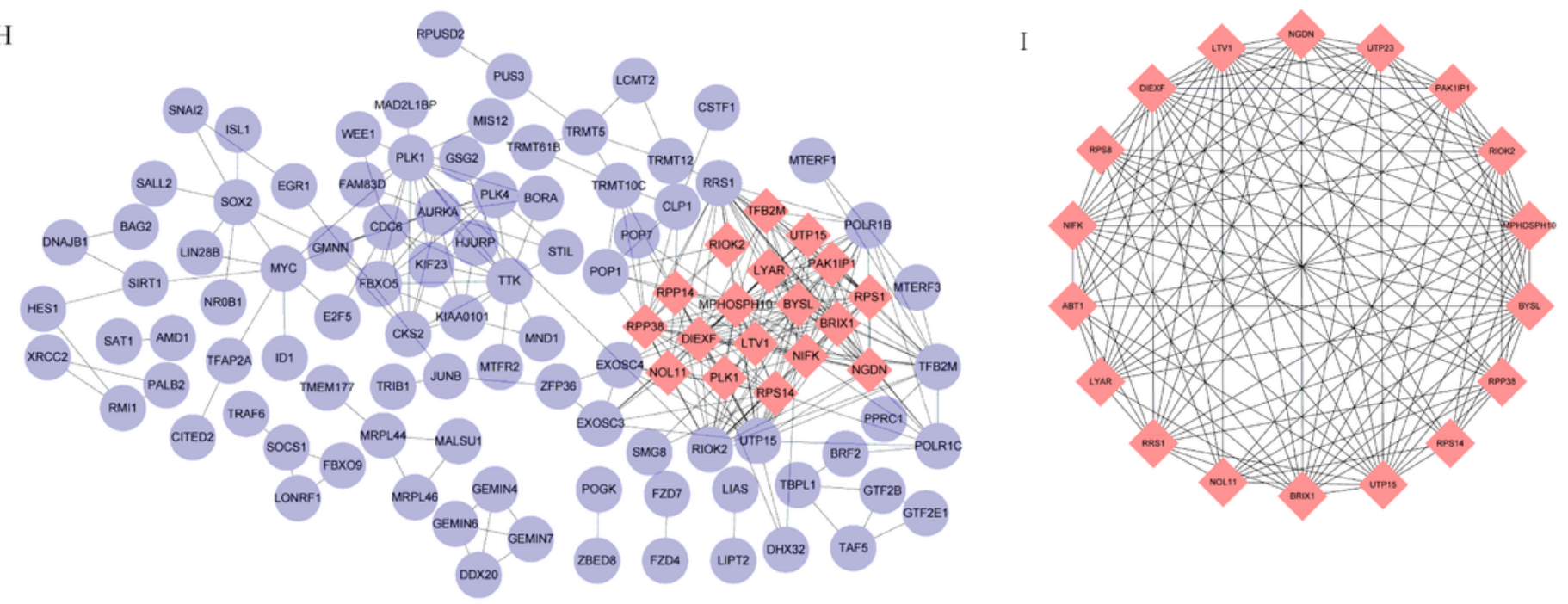

Figure 1

Bioinformatics analysis for gene expression profile Box plots of gene expression profile before (a) and after (b) normalization. Volcano plots of differentially expressed genes(DEGs) (c). GO terms and KEGG pathway enrichment analyses of DEGs were as follows: Biological process(d), Cellular component(e), Molecular function(f), KEGG pathway(g). Dot sizes indicate enriched DEGs counts, while dot colors signify negative log10(p-value). The protein-protein interaction (PPI) network of DEGs was developed by Cytoscape (h), and the most significant module consisted of 18 nodes, and 109 edges was acquired from the PPI network (i) 

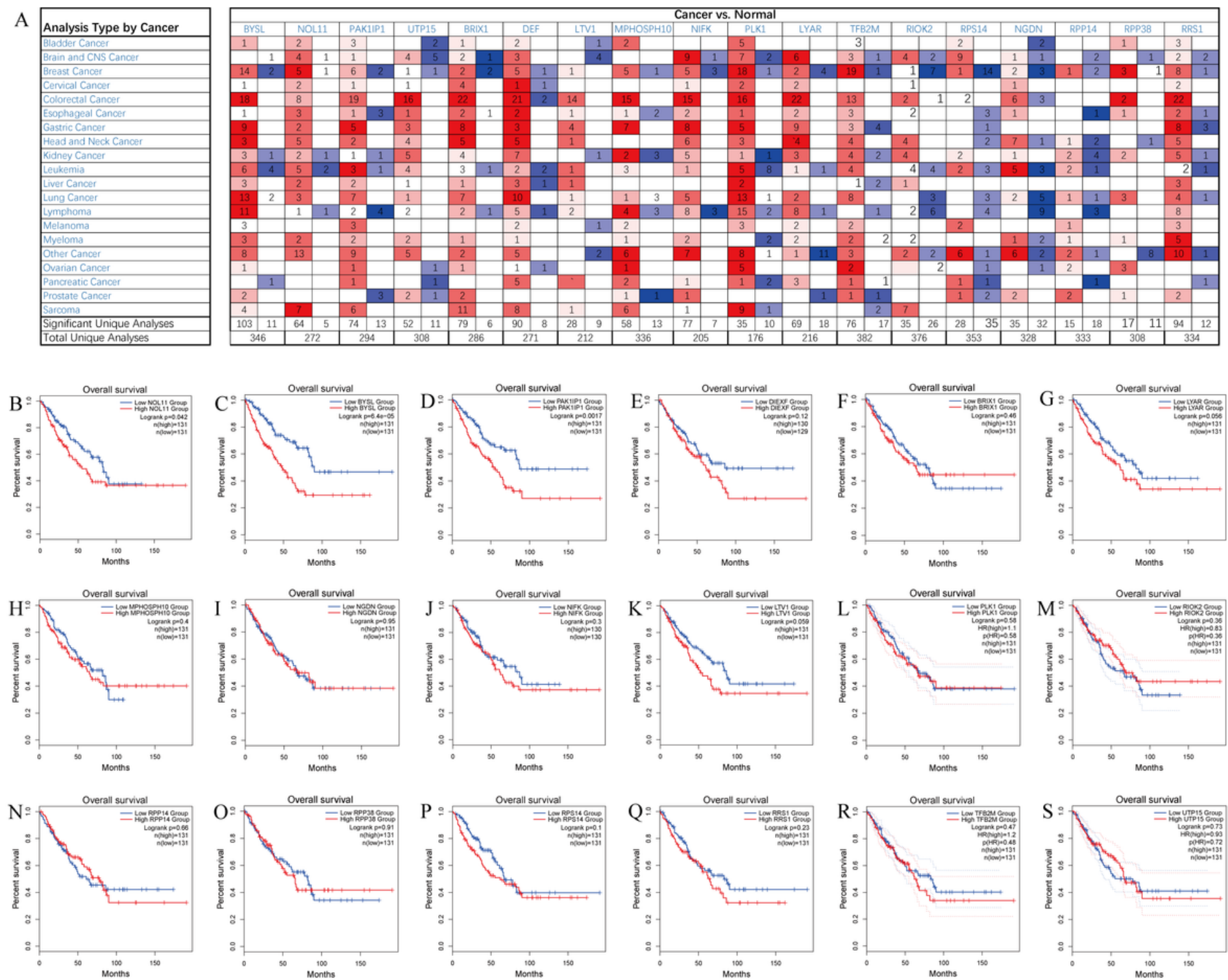

\section{Figure 2}

Validation of hub gene expression by Oncomine and GEPIA2 The summary of the top 18 hub genes in sarcoma(a). Differential analysis of mRNA expression between sarcoma and normal samples was visualized in the Oncomine database. The number in the colored sheets indicates the number of analyses meeting the above thresholds. Gene rank determines the color shade. The red sheets manifest that the mRNA levels of hub genes are higher in sarcoma tissues than in normal samples, while blue sheets represent that the mRNA levels of hub genes are lower in sarcoma samples than in normal samples. The relationship between the top 18 hub genes (b) and OS of patients with sarcoma was analyzed by GEPIA2 using the Kaplan-Meier method and log-rank test 



ESTIMATE - posalive † negative

ESTIMATE + postivo - negative

\section{Figure 3}

Validation of hub gene expression by PCR and immunohistochemistry NOL11, BYSL, and PAK1IP1 mRNA expression levels of A-673 cells and human mesenchymal stem cells, based on quantitative real-time PCR data (a). IHC staining of PAK1IP1, NOL11, and BYSL in Ewing sarcoma tissues ( $\mathrm{n}=22)$. PAK1IP1negative staining (b, c); NOL11-negative staining (d, e); NOL11-positive staining (f, g); BYSL-negative 
staining $(\mathrm{h}, \mathrm{i})$; BYSL-positive staining $(\mathrm{j}, \mathrm{k})$. Magnification $\times 200$ and $\times 400$. Overall survival according to NOL11(I) and BYSL(m) immunostaining in Ewing sarcoma patients 